\title{
Vastus medialis cross-sectional area is positively associated with patella cartilage and bone volumes in a pain-free community-based population
}

\author{
Patricia A Berry ${ }^{1}$, Andrew J Teichtahl1, Ana Galevska-Dimitrovska ${ }^{1}$, Fahad S Hanna ${ }^{1,2}$, \\ Anita E Wluka ${ }^{1,2}$, Yuanyuan Wang ${ }^{1}$, Donna M Urquhart ${ }^{1}$, Dallas R English ${ }^{3,4}$, Graham G Giles ${ }^{3}$ and \\ Flavia M Cicuttini ${ }^{1}$
}

\author{
1'Department of Epidemiology and Preventive Medicine, Monash University Central and Eastern Clinical School, Commercial Road, Melbourne, 3004, \\ Australia \\ 2Baker Heart Research Institute, Commercial Road, Melbourne, 3004, Australia \\ ${ }^{3}$ Cancer Epidemiology Centre, The Cancer Council of Victoria, Rathdowne Street, Melbourne, 3053, Australia \\ ${ }^{4}$ School of Population Health, The University of Melbourne, Bouverie Street, Melbourne, 3010, Australia
}

Corresponding author: Flavia M Cicuttini, Flavia.Cicuttini@med.monash.edu.au

Received: 1 Aug 2008 Revisions requested: 29 Sep 2008 Revisions received: 20 Nov 2008 Accepted: 15 Dec 2008 Published: 15 Dec 2008

Arthritis Research \& Therapy 2008, 10:R143 (doi:10.1186/ar2573)

This article is online at: http://arthritis-research.com/content/10/6/R143

(c) 2008 Berry et al.; licensee BioMed Central Ltd.

This is an open access article distributed under the terms of the Creative Commons Attribution License (http://creativecommons.org/licenses/by/2.0), which permits unrestricted use, distribution, and reproduction in any medium, provided the original work is properly cited.

\begin{abstract}
Introduction Although vastus medialis and lateralis are important determinants of patellofemoral joint function, their relationship with patellofemoral joint structure is unknown. The aim of this study was to examine potential determinants of vastus medialis and lateralis cross-sectional areas and the relationship between the cross-sectional area and patella cartilage and bone volumes.

Methods Two hundred ninety-seven healthy adult subjects had magnetic resonance imaging of their dominant knee. Vastus medialis and lateralis cross-sectional areas were measured 37.5 $\mathrm{mm}$ superior to the quadriceps tendon insertion at the proximal pole of the patella. Patella cartilage and bone volumes were measured from these images. Demographic data and participation in vigorous physical activity were assessed by questionnaire.
\end{abstract}

Results The determinants of increased vastus medialis and lateralis cross-sectional areas were older age $(P \leq 0.002)$, male gender $(P<0.001)$, and greater body mass index $(P \leq 0.07)$. Participation in vigorous physical activity was positively associated with vastus medialis cross-sectional area (regression coefficient [beta] 90.0; 95\% confidence interval [Cl] $38.2,141.7)(P<0.001)$ but not with vastus lateralis crosssectional area (beta $10.1 ; 95 \% \mathrm{Cl}-18.1,38.3)(P=0.48)$. The cross-sectional area of vastus medialis only was positively associated with patella cartilage volume (beta $0.6 ; 95 \% \mathrm{Cl} 0.23$, $0.94)(P=0.001)$ and bone volume (beta $3.0 ; 95 \% \mathrm{Cl} 1.40$, 4.68) $(P<0.001)$ after adjustment for potential confounders.

Conclusions Our results in a pain-free community-based population suggest that increased cross-sectional area of vastus medialis, which is associated with vigorous physical activity, and increased patella cartilage and bone volumes may benefit patellofemoral joint health and reduce the long-term risk of patellofemoral pathology.

\section{Introduction}

Vastus medialis and lateralis are recognised as important determinants of patellofemoral joint function. In particular, these muscles help prevent excessive patella displacement and contribute to patellofemoral joint congruency and stability [1]. Deficiencies in neuromotor control, such as delayed acti- vation of vastus medialis, have been linked to patellofemoral pain syndrome [2] and are speculated to contribute to the risk of patellofemoral subluxation/dislocation [3].

Muscle cross-sectional area, which can be reliably measured via magnetic resonance imaging (MRI) [4] or computerised 
tomography [5], is representative of the force-producing capability of a muscle [6]. Previous studies have examined the cross-sectional area of the quadriceps and have used the midthigh level to assess the maximum force-producing capability of the quadriceps muscle group $[5,7,8]$. Studies examining spinal pathology have demonstrated that reduced cross-sectional area of local muscles that are in close proximity to the joint, attaching directly to the lumbar spine, are associated with low back pain, whereas increased cross-sectional area of these muscles contributes to spinal control [9-11]. However, it is unclear whether an increased cross-sectional area of local muscles at other anatomical sites confers similar benefits. Although distal vastus medialis and lateralis muscles have been implicated in patellofemoral pain [2] and subluxation/dislocation [3], their association with patellofemoral joint structures is unclear. Similarly, the determinants of vastus medialis and lateralis cross-sectional areas are unclear.

In a community-based population of women, we recently showed that increased vastus medialis cross-sectional area was positively associated with patella bone volume, but not patella cartilage volume [12]. Whether this relationship is similar in males is unknown. Moreover, whether the lack of a relationship with cartilage was a true effect or was due to inadequate power of that study is unknown. Thus, the aim of this study was to examine the determinants of vastus medialis and lateralis cross-sectional areas and whether vastus medialis cross-sectional area is associated with patella cartilage volume. In addition, we aimed to determine whether these findings are similar in males and females.

\section{Materials and methods Subjects}

Subjects were recruited from an existing cohort - the Melbourne Collaborative Cohort Study (MCCS), a prospective cohort study of community-based people 40 to 69 years old at recruitment (1990 to 1994) - with the aim of examining the role of lifestyle and genetic factors in the risk of cancer and chronic diseases from middle age and beyond, as previously described [13]. Individuals were excluded if in the last 5 years they had knee pain lasting for more than 24 hours, a previous knee injury requiring non-weight-bearing treatment for more than 24 hours or surgery (including arthroscopy), or a history of any arthritis diagnosed by a medical practitioner. A further exclusion criterion was a contraindication to MRI. The study was approved by The Cancer Council Victoria's Human Research Ethics Committee and the Standing Committee on Ethics in Research Involving Humans of Monash University (Melbourne, Australia). All participants gave written informed consent.

\section{Data collection}

Height and weight were measured and body mass index (BMI) was calculated. At MCCS recruitment from 1990 to 1994, subjects completed a questionnaire that collected demo- graphic and physical activity information. Subjects were asked, 'On average (over the last 6 months), how many times a week did you exercise vigorously for a period of 20 minutes?' Frequency of weekly episodes of vigorous physical activity was categorised into three groups: never, once or twice per week, and at least three times per week. Subjects were categorised as participating in vigorous physical activity if they exercised once or twice per week or at least three times per week. Vigorous was defined as activity leading to sweating or dyspnoea, and examples such as swimming, tennis, netball, athletics (which may involve running, walking, throwing or jumping), and running were listed.

\section{Magnetic resonance imaging}

MRI was performed on the dominant knee as previously described [14]. The following sequence and parameters were used: a T1-weighted fat-suppressed three-dimensional gradient recall acquisition in the steady state; flip angle 55 degrees; repetition time 58 milliseconds; echo time 12 milliseconds; field of view $16 \mathrm{~cm}$; 60 partitions; 512 (frequency direction, superior-inferior) $\times 512$ (phase-encoding direction, anteriorposterior) matrix; one acquisition, time 11 minutes $56 \mathrm{sec}$ onds. Sagittal images were obtained at a partition thickness of $1.5 \mathrm{~mm}$ and an in-plane resolution of $0.31 \mathrm{~mm} \times 0.31 \mathrm{~mm}$ $(512 \times 512$ pixels). The sagittal MR images were reformatted in the axial plane with a partition thickness of $1.25 \mathrm{~mm}$ and an in-plane resolution of $0.31 \times 0.31 \mathrm{~mm}(512 \times 512$ pixels $)$.

\section{Vastus medialis and lateralis cross-sectional areas}

Distal vastus medialis and lateralis cross-sectional areas were measured directly from axial images by one trained observer manually drawing disarticulation contours around the muscle boundaries using the independent workstation software Osiris (Digital Imaging Unit, University Hospital of Geneva, Geneva, Switzerland). The cross-sectional area was measured at the MR slice $37.5 \mathrm{~mm}$ superior to the quadriceps tendon insertion at the proximal pole of the patella, orthogonal to the long axis of the leg. This slice was chosen as it was the largest slice visible across all subjects. The intraobserver reliability for vastus muscle cross-sectional area (expressed as intraclass correlation coefficient) was 0.99 .

\section{Measurement of patella cartilage and bone volumes}

Patella cartilage and bone volumes were determined by manually drawing disarticulation contours around the patella boundaries on images $1.5 \mathrm{~mm}$ apart on sagittal views, using image processing on an independent workstation using the Osiris software as previously described [14,15]. The coefficients of variation were $2.1 \%$ for patella cartilage volume and $2.2 \%$ for patella bone volume [16].

\section{Statistical analyses}

Outcome variables, including distal vastus medialis and lateralis cross-sectional areas and cartilage and bone volumes, were initially assessed for normality and were found to approx- 
imate the normal distribution. Univariable and multiple linear regression models were used to examine determinants of vastus medialis and lateralis cross-sectional areas and the relationship with patella cartilage and bone volumes. Potential confounders, including age, gender, BMl, and participation in vigorous physical activity, were adjusted for in multivariate analyses. $R^{2}$ values were calculated to determine the proportion of variance explained by the multiple regression equation. All analyses were performed using the SPSS statistical package (standard version 14.0; SPSS Inc., Chicago, IL, USA). A $P$ value of less than 0.05 was considered statistically significant.

\section{Results}

The characteristics of the 297 subjects (63\% women) who participated in the study are presented in Table 1.

\section{Determinants of distal vastus medialis and lateralis muscle cross-sectional areas}

The determinants of vastus medialis and lateralis cross-sectional areas are presented in Table 2. After adjustment for potential confounders, including gender, BMI, and participation in vigorous physical activity, we found that age was negatively associated with vastus medialis and with vastus lateralis cross-sectional area. Women had significantly smaller vastus medialis and vastus lateralis cross-sectional area than men in multivariate analysis. BMI was positively associated with vastus medialis cross-sectional area in multivariate analysis, with a similar trend observed with vastus lateralis. Participation in vigorous physical activity (binary variable) was positively associated with vastus medialis cross-sectional area in multivariate analysis, whereas no significant association was seen for vastus lateralis. In multivariate analysis, similar results were obtained when the relationship between increasing frequency of weekly episodes of vigorous physical activity and vastus medialis (beta 54.9; 95\% confidence interval [Cl] 24.6, 85.2)
$(P=0.0004)$ and vastus lateralis (beta $3.0 ; 95 \% \mathrm{Cl}-13.6$, 19.5) $(P=0.73)$ was considered.

\section{Relationship between distal vastus medialis and lateralis cross-sectional areas and patella structures}

The association between vastus medialis and lateralis crosssectional areas and patella structures, including cartilage and bone volumes, is presented in Table 3 . Vastus medialis, but not vastus lateralis, cross-sectional area was positively associated with patella cartilage volume and bone volume in multivariate analysis. To determine whether gender differences in effect exist, men and women were analysed separately in multivariate analyses. The relationship between vastus medialis cross-sectional area and patella cartilage volume was similar for men (beta $0.68 ; 95 \% \mathrm{Cl}-0.01,1.38)(P=0.054)$ and women (beta $0.38 ; 95 \% \mathrm{Cl}-0.02,0.77)(P=0.06)$, and the relationship between vastus medialis cross-sectional area and patella bone volume was also similar for men (beta 3.79; 95\% $\mathrm{Cl} 0.95,6.63)(P=0.01)$ and women (beta $2.16 ; 95 \% \mathrm{Cl}$ $0.07,4.24)(P=0.04)$. Similar results were obtained when height and weight were used in the multivariate regression equation instead of BMl (data not shown).

\section{Discussion}

Our results have demonstrated that older age, female gender, and lower BMl are associated with reduced vastus medialis and lateralis cross-sectional areas. Participation in vigorous physical activity was associated with increased vastus medialis, but not vastus lateralis, cross-sectional area. Similarly, only increased vastus medialis cross-sectional area was associated with increased patella cartilage and bone volumes. These findings suggest that increased vastus medialis cross-sectional area may benefit patellofemoral joint health.

Our measure of vastus medialis and lateralis cross-sectional areas demonstrated expected relationships, with age and female gender being associated with a reduction in vastus

Table 1

Characteristics of the study population $(n=297)$

\begin{tabular}{lr}
\hline & Mean (SD)a \\
\hline Age at magnetic resonance imaging, years & $59.1(6.3)$ \\
Females, number (percentage) & $186(63)$ \\
Body mass index, $\mathrm{kg} / \mathrm{m}^{2}$ & $25.2(3.8)$ \\
Participation in vigorous physical activity, number of subjects (percentage) ${ }^{\mathrm{b}}$ & $107(36)$ \\
Distal vastus medialis cross-sectional area, $\mathrm{mm}^{2}$ & $1,171(306)$ \\
Distal vastus lateralis cross-sectional area, $\mathrm{mm}^{2}$ & $333(127)$ \\
Patella cartilage volume, $\mathrm{mm}^{3}$ & $2,656(886)$ \\
Patella bone volume, $\mathrm{mm}^{3}$ & $20,276(4,667)$ \\
\hline
\end{tabular}

aValues are reported as mean (standard deviation, SD) unless otherwise stated. bParticipation in vigorous physical activity at least once or twice a week or at least three times per week (over the last 6 months) for a period of 20 minutes that leads to sweating or dyspnoea. 
Table 2

Determinants of distal vastus medialis and vastus lateralis cross-sectional areas

\begin{tabular}{|c|c|c|c|}
\hline & $\begin{array}{l}\text { Univariate regression coefficient } \\
\qquad(95 \% \mathrm{Cl})\end{array}$ & $\begin{array}{l}\text { Multivariate regression coefficient } \\
\qquad(95 \% \mathrm{Cl})^{\mathrm{a}}\end{array}$ & $R^{2}$ for model ${ }^{\mathrm{a}}$ \\
\hline \multicolumn{4}{|l|}{ Vastus medialis cross-sectional area, $\mathrm{mm}^{2}$} \\
\hline Age, years & $-1.39(-7.78,4.99)$ & $-7.1(-11.7,-2.6)^{b}$ & 0.532 \\
\hline Gender & $-415.9(-470.2,-361.5)^{b}$ & $-391.6(-443.0,-340.2)^{b}$ & 0.532 \\
\hline Body mass index, $\mathrm{kg} / \mathrm{m}^{2}$ & $27.5(18.9,36.2)^{b}$ & $21.7(15.2,28.2)^{\mathrm{b}}$ & 0.532 \\
\hline $\begin{array}{l}\text { Participation in vigorous physical activity, } \\
\text { yes/no }\end{array}$ & $118.6(47.0,190.1)^{b}$ & $90.0(38.2,141.7)^{b}$ & 0.532 \\
\hline \multicolumn{4}{|l|}{ Vastus lateralis cross-sectional area, $\mathrm{mm}^{2}$} \\
\hline Age, years & $-1.7(-4.4,0.94)$ & $-3.3(-5.8,-0.8)^{b}$ & 0.192 \\
\hline Gender & $-105.6(-133.0,-78.2)^{b}$ & $-106.0(-134.0,-78.0)^{b}$ & 0.192 \\
\hline Body mass index, $\mathrm{kg} / \mathrm{m}^{2}$ & $4.8(1.1,8.6)^{c}$ & $3.3(-0.2,6.8)$ & 0.192 \\
\hline $\begin{array}{l}\text { Participation in vigorous physical activity, } \\
\text { yes/no }\end{array}$ & $23.2(-6.8,53.3)$ & $10.1(-18.1,38.3)$ & 0.192 \\
\hline
\end{tabular}

aAge, gender, body mass index, and participation in vigorous physical activity included in multivariate regression equation. ${ }^{\mathrm{b}} P<0.01 .{ }^{\mathrm{c}} P<0.05$. $\mathrm{Cl}$, confidence interval.

medialis and lateralis cross-sectional areas, whereas BMI was associated with greater vastus medialis and lateralis crosssectional areas. Though not examined specifically in this study, a reduction in muscle size has been shown to be common with aging $[4,17,18]$, and it has been shown that females have, on average, less muscle mass relative to males $[17,19]$. In contrast, a larger BMI may be inherently associated with increased muscle cross-sectional area due to a larger body size. We have also demonstrated that participation in vigorous physical activity was associated with increased vastus medialis, but not vastus lateralis, cross-sectional area. A primary action of the distal portion of vastus medialis is to dynamically restrain the natural tendency of the patella to track laterally [1]. Therefore, the relationship between vastus medialis cross-sectional area and participation in physical activity may, in the setting of vigorous physical activity, reflect vastus medialis hypertrophy in an attempt to restrain excessive lateral patella displacement.
We have previously examined the relationship between vastus medialis and lateralis cross-sectional areas and patella structures [12] in an independent and smaller $(n=175)$ cohort of healthy women (mean age of 52 years). In this previous study, we demonstrated a positive association between vastus medialis cross-sectional area and patella bone volume, but not cartilage volume. In the present study, we examined an entirely independent cohort and substantiated all previously significant findings while also noting that increased patella cartilage volume is significantly associated with an increased crosssectional area of vastus medialis. The discrepancy in cartilage volume results between the two independent studies may be attributable to the smaller sample size of our previous study. Moreover, in the present study, we included males and thus expanded the generalisability of our findings. Subsequently, the present study substantiates previous findings pertaining to both men and women. In the past and present studies, we

Table 3

Relationship between distal vastus medialis and vastus lateralis cross-sectional areas and patella structures

\begin{tabular}{|c|c|c|c|}
\hline & Univariate regression coefficient $(95 \% \mathrm{Cl})$ & Multivariate regression coefficient $(95 \% \mathrm{Cl})$ & $R^{2}$ for model \\
\hline \multicolumn{4}{|c|}{ Vastus medialis cross-sectional area, $\mathrm{mm}^{2}$} \\
\hline Patella cartilage volume, $\mathrm{mm}^{3}$ & $1.5(1.3,1.8)^{a}$ & $0.58(0.23,0.94)^{a}$ & $0.472^{b}$ \\
\hline Patella bone volume, $\mathrm{mm}^{3}$ & $9.09(7.69,10.50)^{a}$ & $3.04(1.40,4.68)^{a}$ & $0.592^{c}$ \\
\hline \multicolumn{4}{|c|}{ Vastus lateralis cross-sectional area, $\mathrm{mm}^{2}$} \\
\hline Patella cartilage volume, $\mathrm{mm}^{3}$ & $1.67(0.89,2.44)^{a}$ & $-0.33(-0.99,0.33)$ & $0.455^{b}$ \\
\hline Patella bone volume, $\mathrm{mm}^{3}$ & $10.3(6.19,14.31)^{\mathrm{a}}$ & $-1.31(-4.40,1.78)$ & $0.574^{\mathrm{c}}$ \\
\hline
\end{tabular}

a $P<0.01$. bAge, gender, body mass index, and participation in vigorous physical activity and patella cartilage volume included in the multivariate regression equation. 'Age, gender, body mass index, and participation in vigorous physical activity and patella bone volume included in the multivariate regression equation. $\mathrm{Cl}$, confidence interval. 
have found vastus medialis, rather than vastus lateralis, to be the significant determinant of patella structures. The relative importance of vastus medialis at the patellofemoral joint is also supported by electromyography studies, which demonstrated that delayed activation of vastus medialis relative to vastus lateralis is associated with patellofemoral pathologies, including pain [2] and subluxation/dislocation [3].

How a greater vastus medialis cross-sectional area mediates increased patella cartilage and bone volumes is unclear. Previous studies have shown that reduced cross-sectional areas of local spinal muscles are associated with instability and low back pain [9-11]. As indicated by extrapolating such data to the patellofemoral joint, it may be that a greater cross-sectional area of the vastus medialis muscle helps to prevent the natural tendency of the patella to track laterally [1] and thus reduce any shearing damage that may occur to articular surfaces. This, in turn, may produce an optimal biomechanical environment and have a beneficial effect on patella structures, including cartilage and bone. Moreover, our recent longitudinal data have suggested that increased patella bone volume may be advantageous to patellofemoral joint health, as increased baseline bone volume was associated with a reduction in the rate of patella cartilage volume loss [20]. Therefore, it may be that increased vastus medialis cross-sectional area benefits the patellofemoral joint via biomechanical effects on both patella bone and cartilage volumes. Longitudinal studies are required to determine whether increased vastus medialis cross-sectional area reduces the risk of long-term patellofemoral pathology.

We examined a healthy population without knee pain or pathology and our results cannot be generalised to symptomatic populations or those with established knee pathology. Nevertheless, studying a healthy population allows the identification of factors that may be associated with early structural changes at the knee and reduces the confounding effect of reduction in muscle size due to pain-related disuse. Furthermore, this study may have been limited by our method for assessing the cross-sectional area of the distal vastus medialis and lateralis muscles. We measured the vastus muscles at the MR slice $37.5 \mathrm{~mm}$ superior to the quadriceps tendon insertion at the proximal pole of the patella. This slice was chosen as it was the largest slice visible across all subjects. Moreover, we have calculated cross-sectional area rather than the physiological cross-sectional area (PCSA) that previously has been employed to investigate muscle-force relationships [21]. Calculating the PCSA requires imaging of the entire length of the muscle fibre, which would be a costly and timely exercise using MRI. However, our measure of cross-sectional area showed the expected relationships with age, gender, and BMI. It may be hypothesised that people with larger muscles inherently have larger joint structures, including cartilage and bone volumes, but if this were the case, vastus lateralis would have been significantly associated with patella cartilage and bone.
Moreover, the relationships observed in this study were independent of $\mathrm{BMI}$, height, and weight (data not shown) as a measure of body size. Although the determinants of patella structure remain unclear, there is likely to be a complex interplay between the surrounding soft tissue support (for example, vastus medialis and lateralis muscles) and the bony geometry (for example, femoral sulcus angle and patella tilt). Therefore, future studies may benefit from examining other articular supports that may also contribute to patellofemoral joint structure and function.

\section{Conclusion}

Our results in a pain-free population without clinical knee osteoarthritis suggest that increased cross-sectional area of vastus medialis, which is associated with participation in vigorous physical activity, and increased patella cartilage and bone volumes, may benefit patellofemoral joint health. This warrants further investigation as a potential method for reducing longterm patellofemoral pathology.

\section{Competing interests}

The authors declare that they have no competing interests.

\section{Authors' contributions}

FMC and AEW participated in the design of the study and in the analysis and interpretation of data and reviewed the manuscript. DRE and GGG participated in the acquisition of data and in the analysis and interpretation of data and reviewed the manuscript. FSH participated in carrying out the measurement of muscle structure and in the analysis and interpretation of data and reviewed the manuscript. AG-D participated in carrying out the measurement of muscle structure. YW carried out the measurement of knee cartilage and bone structure, participated in the analysis and interpretation of data, and reviewed the manuscript. PAB and AJT performed the statistical analysis and interpretation of data and drafted the manuscript. DMU participated in the analysis and interpretation of data and reviewed the manuscript. All authors read and approved the final manuscript.

\section{Acknowledgements}

The Melbourne Collaborative Cohort Study recruitment was funded by VicHealth and The Cancer Council of Victoria. This study was funded by a program grant from the National Health and Medical Research Council (NHMRC) (209057) and was further supported by infrastructure provided by The Cancer Council of Victoria. We would like to acknowledge the NHMRC (project grant 334150) and the Colonial Foundation. AEW, YW, and DMU are the recipients of NHMRC Public Health Fellowships ( 317840,465142 , and 284402 , respectively). PAB is the recipient of an Australian Post-graduate Award PhD Scholarship. We would especially like to thank the study participants, who made this study possible.

\section{References}

1. Elias DA, White LM: Imaging of patellofemoral disorders. Clin Radiol 2004, 59:543-557.

2. Cowan SM, Bennell KL, Hodges PW, Crossley KM, McConnell J: Delayed onset of electromyographic activity of vastus medialis obliquus relative to vastus lateralis in subjects with patel- 
lofemoral pain syndrome. Arch Phys Med Rehabil 2001, 82:183-189.

3. Gilleard W, McConnell J, Parsons D: The effect of patellar taping on the onset of vastus medialis obliquus and vastus lateralis muscle activity in persons with patellofemoral pain. Phys Ther 1998, 78:25-32.

4. Frontera WR, Hughes VA, Fielding RA, Fiatarone MA, Evans WJ, Roubenoff R: Aging of skeletal muscle: a 12-yr longitudinal study. J Appl Physio/ 2000, 88:1321-1326.

5. Sipila S, Elorinne M, Alen M, Suominen H, Kovanen V: Effects of strength and endurance training on muscle fibre characteristics in elderly women. Clin Physiol 1997, 17:459-474.

6. Bamman MM, Newcomer BR, Larson-Meyer DE, Weinsier RL, Hunter GR: Evaluation of the strength-size relationship in vivo using various muscle size indices. Med Sci Sports Exerc 2000, 32:1307-1313.

7. Narici MV, Hoppeler H, Kayser B, Landoni L, Claassen H, Gavardi C, Conti M, Cerretelli P: Human quadriceps cross-sectional area, torque and neural activation during 6 months strength training. Acta Physiol Scand 1996, 157:175-186.

8. Harridge SD, Kryger A, Stensgaard A: Knee extensor strength, activation, and size in very elderly people following strength training. Muscle Nerve 1999, 22:831-839.

9. Hides JA, Stokes MJ, Saide M, Jull GA, Cooper DH: Evidence of lumbar multifidus muscle wasting ipsilateral to symptoms in patients with acute/subacute low back pain. Spine 1994, 19:165-172.

10. Danneels LA, Vanderstraeten GG, Cambier DC, Witvrouw EE, De Cuyper HJ: CT imaging of trunk muscles in chronic low back pain patients and healthy control subjects. Eur Spine J 2000, 9:266-272.

11. Hides J, Gilmore C Stanton W, Bohlscheid E, Hides J, Gilmore C, Stanton W, Bohlscheid E: Multifidus size and symmetry among chronic LBP and healthy asymptomatic subjects. Manual Therapy 2008, 13:43-49.

12. Berry PA, Hanna FS, Teichtahl AJ, Wluka AE, Urquhart DM, Bell RJ, Davis SR, Cicuttini FM: Vastus medialis cross-sectional area is associated with patella cartilage defects and bone volume in healthy women. Osteoarthritis Cartilage 2008, 16:956-960.

13. Giles GG, English DR: The Melbourne Collaborative Cohort Study. IARC Sci Pub/ 2002, 156:69-70.

14. Cicuttini F, Forbes A, Morris K, Darling S, Bailey M, Stuckey S: Gender differences in knee cartilage volume as measured by magnetic resonance imaging. Osteoarthritis Cartilage 1999, 7:265-271.

15. Wluka AE, Davis SR, Bailey M, Stuckey SL, Cicuttini FM: Users of oestrogen replacement therapy have more knee cartilage than non-users. Ann Rheum Dis 2001, 60:332-336.

16. Teichtahl AJ, Jackson BD, Morris ME, Wluka AE, Baker R, Davis SR, Cicuttini FM: Sagittal plane movement at the tibiofemoral joint influences patellofemoral joint structure in healthy adult women. Osteoarthritis Cartilage 2006, 14:331-336.

17. Frontera WR, Hughes VA, Lutz KJ, Evans WJ: A cross-sectional study of muscle strength and mass in 45- to 78-yr-old men and women. J Appl Physiol 1991, 71:644-650.

18. Cohn SH, Vartsky D, Yasumura S, Sawitsky A, Zanzi I, Vaswani A, Ellis KJ: Compartmental body composition based on total-body nitrogen, potassium, and calcium. Am J Physiol 1980, 239:E524-530.

19. Vandervoort AA, McComas AJ: Contractile changes in opposing muscles of the human ankle joint with aging. J Appl Physiol 1986, 61:361-367.

20. Wijayaratne SP, Teichtahl AJ, Wluka AE, Hanna F, Bell R, Davis SR, Adams J, Cicuttini FM: The determinants of change in patella cartilage volume - a cohort study of healthy middleaged women. Rheumatology (Oxford) 2008, 47:1426-1429.

21. Fukunaga T, Roy RR, Shellock FG, Hodgson JA, Day MK, Lee PL, Kwong-Fu H, Edgerton VR: Physiological cross-sectional area of human leg muscles based on magnetic resonance imaging. J Orthop Res 1992, 10:928-934. 